\title{
Incomplete Information Choice on Incumbents, Cognitive Ability and Personality
}

\author{
Sergio Da Silva1 ${ }^{*}$, Raul Matsushita2 ${ }^{2}$, Mauricio Ramos ${ }^{1}$ \\ ${ }^{1}$ Department of Economics, Federal University of Santa Catarina, Florianopolis, Brazil \\ ${ }^{2}$ Department of Statistics, University of Brasilia, Brasilia, Brazil \\ Email: * professorsergiodasilva@gmail.com
}

How to cite this paper: Da Silva, S., Matsushita, R. and Ramos, M. (2019) Incomplete Information Choice on Incumbents, Cognitive Ability and Personality. Open Access Library Journal, 6: e5476.

https://doi.org/10.4236/oalib.1105476

Received: May 17, 2019

Accepted: June 2, 2019

Published: June 5, 2019

Copyright () 2019 by author(s) and Open Access Library Inc.

This work is licensed under the Creative Commons Attribution International License (CC BY 4.0).

http://creativecommons.org/licenses/by/4.0/

\begin{abstract}
In choices under incomplete information on incumbents, consumers with stronger preferences are more likely to reinforce their prior choices with motivated reasoning. However, in situations where incomplete information is restricted only to the prior choice, consumers with stronger preferences are more likely to abandon, not reinforce, their prior choices due to cognitive dissonance. Here, we consider how cognitive ability and personality traits mediate such interplay between motivated reasoning and cognitive dissonance. We set an experiment to show that consumers with a stronger System 2 are more likely to engage in motivated reasoning to reinforce the prior choice and thus suffer less cognitive dissonance. Cognitive dissonance can, however, overcome motivated reasoning for those cognitively poor consumers who are more emotional, less humble, less extraverted and less conscientious.
\end{abstract}

\section{Subject Areas \\ Behavioral Economics, Consumer Behavior}

\section{Keywords}

Choice under Incomplete Information, Prior Choices, Preference Strength, Cognitive Dissonance, Motivated Reasoning, Cognitive Reflection, HEXACO Personality Model, Behavioral Economics

\section{Introduction}

Consumers often make choices between options with missing information and then come across second choices that have more complete information than that were presented in the prior choice [1]. As a result, they have to decide between the option chosen in the prior choice and the new one with more information. 
Research on choice under incomplete information on incumbents has found that consumers often engage in motivated reasoning [2] [3] in order to process missing information, preferentially in a confirmatory way [4]. Thus, those with relatively stronger preferences for their prior choice should be more likely to have a motivated reasoning to reinforce it, reducing the likelihood of abandoning the option chosen.

However, it has been suggested recently [5] that in situations where incomplete information is restricted only to the prior choice, it is unlikely that consumers will reinforce it. This happens due to cognitive dissonance [6]: The preliminary positive attitude toward the option chosen is accompanied by a negative affect thereafter. Those with relatively stronger prior preferences experience more cognitive dissonance when information on new attributes is missing on just the incumbent, but not on its competitors.

Thus, the conventional wisdom has been reversed, and the issue of how preference strength affects consumers' reactions to missing information on their prior choice is unsettled.

Two contrasting forces are at the heart of this debate: motivated reasoning and cognitive dissonance. When consumers are strongly engaged in motivated reasoning, this can override the affective process guiding their abandonment of the incumbent. In such a situation, they are less likely to abandon their prior choice. The reverse occurs when motivated reasoning is weaker than the affective reaction leading to cognitive dissonance, where consumers are more likely to abandon their prior choice.

Here, we set an experiment similar to Study 1 of Irmak and colleagues [5] to further consider a way to gauge both motivated reasoning and cognitive dissonance. In this connection, we administer a cognitive reflection test [7] and a HEXACO's personality assessment [8] of the participants in our experiment. Irmak and coauthors recruited a sample of 153 participants from Amazon's Mechanical Turk, and this very fact suggests a self-selection bias toward volunteers who are more likely to be open to new experience. Here, we explicitly consider such a personality trait (and others) in a sample that is probably not so exposed to self-selection.

The two-mind approach in cognitive psychology [9] [10] posits that two systems compete for control of judgments and choices. Intuitive decisions that require little reflection use "System 1". Decisions that use mental models or simulations of future possibilities engage "System 2". System 1 is composed of a set of autonomous subsystems that comprise input modules related to specific-domain knowledge. System 2 is related to abstract reasoning and the way we think using hypotheses. A simple test can measure how individuals differ in cognitive ability in terms of the relative powers of their System 1 and System 2: the cognitive reflection test (CRT) [7]. Individuals scoring higher on the CRT display enhanced ability for using their System 2 to override System 1 proclivities. Here, we hypothesize that those with a stronger System 2 are more likely to develop motivated reasoning to reinforce their prior choice and thus to suffer less cognitive dis- 
sonance.

Differences in behavior can be predicted not only by cognitive ability and intelligence, but also by other Big Five personality traits: emotionality (E), extraversion (X), agreeableness (A), conscientiousness (C) and openness to experience $(\mathrm{O})$ [11] [12]. A trait of honesty-humility $(\mathrm{H})$ can also be added to the Big Five, and this forms a HEXACO model of six dimensions [13]. The HEXACO can be evaluated with adequate validity by a 24-item inventory [8], explained in detail later.

The rest of this paper is organized as follows: Section 2 describes the experiment and the methods used; Section 3 shows the results found; and Section 4 concludes this study.

\section{Materials and Methods}

We prepared three questionnaires to: 1) measure cognitive ability through the CRT; 2) evaluate personality traits through the brief HEXACO inventory (BHI); 3) examine the mediation of cognitive dissonance in the effect of preference strength on switching. Questionnaire 3 was similar to that in Study 1 of Irmak and coauthors. The three questionnaires were presented following the sequence 1, 2 and 3. The experimenter (M.R.) employed the app Eval \& Go to distribute the questionnaires online and sent an invitation to initial volunteers via WhatsApp, Facebook and Skype. The initial volunteers came from Florianopolis, southern Brazil. Then, he asked the volunteers to resend the link to friends. The questionnaires were sent from March 20, 2018 to April 16, 2018. In total, 404 volunteers participated, but only 233 provided valid responses. The data set is available at Figshare (https://doi.org/10.6084/m9.figshare.7582019.v1). (Ethics and consent: This experiment is part of a larger project that is registered at Plataforma Brasil (Comissão Nacional de Ética em Pesquisa) under No. 64758617.2.0000.0121.)

The cognitive reflection test [7] encompasses three questions that are designed to elicit automatic responses that are compelling, but wrong. CRT

1) A bat and a ball cost $\$ 1.10$ in total. The bat costs $\$ 1.00$ more than the ball. How much does the ball cost? cents

[Correct response: 5; intuitive response: 10]

2) If it takes 5 machines 5 minutes to make 5 widgets, how long would it take 100 machines to make 100 widgets? minutes

[Correct response: 5; intuitive response: 100]

3) In a lake, there is a patch of lily pads. Every day, the patch doubles in size. If it takes 48 days for the patch to cover the entire lake, how long would it take the patch to cover half the lake? days 
[Correct response: 47; intuitive response: 24$]$

The experimenter instructed participants to respond to the three questions in less than 30 seconds. This assured him they made an automatic choice. The appEval \& Go automatically switched to another screen after 30 seconds had passed. He also asked whether each participant already knew one or all of the three questions. Those who reported to know at least one of the questions were redirected by Eval \& Go to an alternative CRT [14], as follows:

CRT (alternative questions)

1) If John can drink one barrel of water in 6 days, and Mary can drink one barrel of water in 12 days, how long would it take them to drink one barrel of water together? days.

[Correct response: 4; intuitive response: 9]

2) Jerry received both the 15th highest and the 15th lowest mark in the class. How many students are in the class? students.

[Correct response: 29; intuitive response: 30]

3) A man buys a pig for $\$ 60$, sells it for $\$ 70$, buys it back for $\$ 80$ and sells it finally for $\$ 90$. How much has he made? dollars.

[Correct response: 20; intuitive response: 10]

As observed, we gauged the personality traits of the participants through the brief HEXACO inventory (BHI) [8]. This questionnaire randomly assesses the traits, and uses a direct or a reverse Likert scale (Table 1). Thus, participants were instructed to indicate to what extent they agreed with the 24 statements below, using: 1 = strongly disagree; 2 = disagree; 3 = neither agree nor disagree; $4=$ agree; 5 = strongly agree.

BHI

1) I can look at a painting for a long time.

2) I make sure that things are in the right spot.

3) I remain unfriendly to someone who was mean to me.

4) Nobody likes talking to me.

5) I am afraid of feeling pain.

6) I find it difficult to lie.

7) I think science is boring.

8) I postpone complicated tasks as long as possible.

9) I often express criticism.

10)I easily approach strangers.

11)I worry less than others.

12)I would like to know how to make lots of money in a dishonest manner.

13)I have a lot of imagination.

14)I work very precisely.

15)I tend to quickly agree with others. 
Table 1. The BHI in detail.

\begin{tabular}{|c|c|c|c|c|c|c|c|}
\hline Personality trait & BHI item & Main characteristic & \multicolumn{5}{|c|}{ Likert scale } \\
\hline \multirow{4}{*}{ Honesty-humility } & 6 & Sincerity & 1 & 2 & 3 & 4 & 5 \\
\hline & $12^{*}$ & Fairness & 5 & 4 & 3 & 2 & 1 \\
\hline & $18^{*}$ & Greed avoidance & 5 & 4 & 3 & 2 & 1 \\
\hline & $24^{*}$ & Modesty & 5 & 4 & 3 & 2 & 1 \\
\hline \multirow{4}{*}{ Emotionality } & 5 & Fearfulness & 1 & 2 & 3 & 4 & 5 \\
\hline & $11^{*}$ & Anxiety & 5 & 4 & 3 & 2 & 1 \\
\hline & $17^{\star}$ & Dependence & 5 & 4 & 3 & 2 & 1 \\
\hline & 23 & Sentimentality & 1 & 2 & 3 & 4 & 5 \\
\hline \multirow{4}{*}{ Extraversion } & $4^{*}$ & Social self-esteem & 5 & 4 & 3 & 2 & 1 \\
\hline & 10 & Social boldness & 1 & 2 & 3 & 4 & 5 \\
\hline & 16 & Sociability & 1 & 2 & 3 & 4 & 5 \\
\hline & $22^{*}$ & Liveliness & 5 & 4 & 3 & 2 & 1 \\
\hline \multirow{4}{*}{ Agreeableness } & $3^{*}$ & Forgiveness & 5 & 4 & 3 & 2 & 1 \\
\hline & $9^{*}$ & Gentleness & 5 & 4 & 3 & 2 & 1 \\
\hline & 15 & Flexibility & 1 & 2 & 3 & 4 & 5 \\
\hline & 21 & Patience & 1 & 2 & 3 & 4 & 5 \\
\hline \multirow{4}{*}{ Conscientiousness } & 2 & Organization & 1 & 2 & 3 & 4 & 5 \\
\hline & $8^{*}$ & Diligence & 5 & 4 & 3 & 2 & 1 \\
\hline & 14 & Perfectionism & 1 & 2 & 3 & 4 & 5 \\
\hline & $20^{*}$ & Prudence & 5 & 4 & 3 & 2 & 1 \\
\hline \multirow{4}{*}{ Openness to experience } & 1 & Aesthetic appreciation & 1 & 2 & 3 & 4 & 5 \\
\hline & $7^{\star}$ & Inquisitiveness & 5 & 4 & 3 & 2 & 1 \\
\hline & 13 & Creativity & 1 & 2 & 3 & 4 & 5 \\
\hline & 19 & Unconventionality & 1 & 2 & 3 & 4 & 5 \\
\hline
\end{tabular}

* Measured considering a reverse Likert scale. Source: [8].

16)I like to talk with others.

17)I can easily overcome difficulties on my own.

18)I want to be famous.

19)I like people with strange ideas.

20)I often do things without really thinking.

21)Even when I'm treated badly, I remain calm.

22)I am seldom cheerful.

23)I have to cry during sad or romantic movies.

24)I am entitled to special treatment.

The second column in Table 1 refers to traits that are shuffled in the previous 24 statements of the BHI. The third column in Table 1 shows that each of the six HEXACO traits is gauged by the scores in four major characteristics. The scores 
in each of the six traits, which can vary from 4 (lowest) to 20 (highest), are averaged through the scores in each of the four characteristics of a trait. Whenever the score of a participant's trait falls below (above) the median, this trait is considered weak (strong) for the participant.

Questionnaire 3 sought to elicit the mediation of cognitive dissonance in the effect of preference strength on switching, by manipulating the preference strength [5]. Participants were asked to imagine buying a laptop computer and came across two options. According to the option chosen in a pretest, those with stronger prior preference strength were presented with two computers described on RAM size ( 4 or $6 \mathrm{~GB}$ ), and number of shipment companies used ( 3 or 5 companies); while, for those with weaker prior preference strength, the RAM size attribute was replaced with a relatively unimportant attribute: the number of different color options (6 or 10). Participants indicated their preference strength in the pretest after choosing one option by using $1=$ very weak, $\ldots, 10=$ very strong.

Participants eventually chose one of the options: Laptop A or Laptop B. Then, an article (supposedly from PC Magazine) was presented on the weight of portable computers, explaining their relation with portability and presenting weight variation in current models $(0.9$ to $1.8 \mathrm{~kg}$ ). The participants were then presented to the same set with the two options, which now contained additional weight information. Specifically, the weight for the option they had previously rejected was listed as $1.6 \mathrm{~kg}$; however, this value was missing for the prior choice. After reading the article and obtaining the new information, participants choose again between the two options: Laptop A or Laptop B.

\section{Results}

From the 233 participants, 22 knew the original CRT questionnaire [7] and thus were redirected to the alternative one [14]. Questions missed were considered incorrect for those who proceeded to the subsequent questionnaires. Figure 1 shows the overall performance on the CRT, while Figure 2 details performance related to the prior choice. Those who picked Laptop B as a prior choice scored less on the CRT. We then expected those participants to make more automatic choices thereafter, by using their System 1.

Figure 3 shows CRT performance related to the second choice, after the participants received new information. Those who picked Laptop A as their final choice outsmarted those who picked Laptop B. Those who preferred Laptop A over Laptop B showed more cognitive ability in the CRT and therefore were more likely to have engaged in motivated reasoning and to fall less prey to cognitive dissonance.

As for the HEXACO personality traits, Table 2 shows an overview. For the overall sample, participants showed a higher degree of honesty-humility, emotionality and conscientiousness, and thus a lower degree of extraversion, agreeableness and openness to experience. 


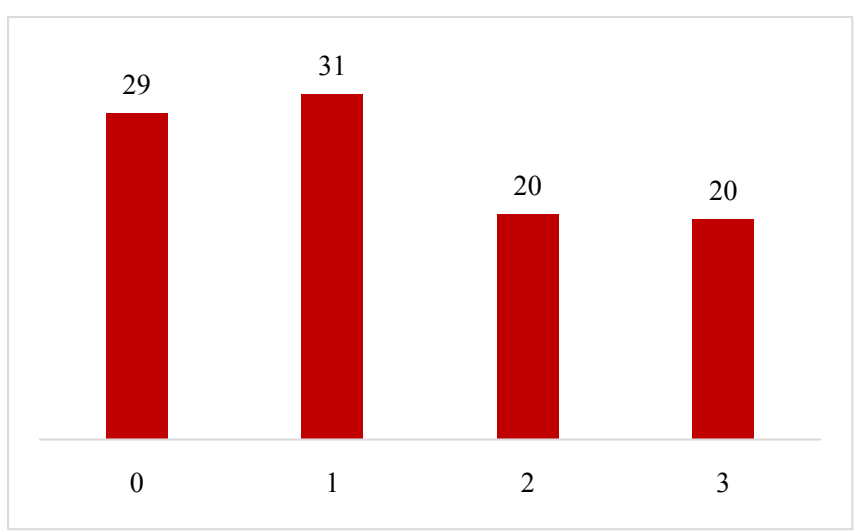

Figure 1. Overall CRT performance (\%).



Figure 2. CRT performance (\%) versus prior choice, Blue font: Laptop A; Red font: Laptop B.

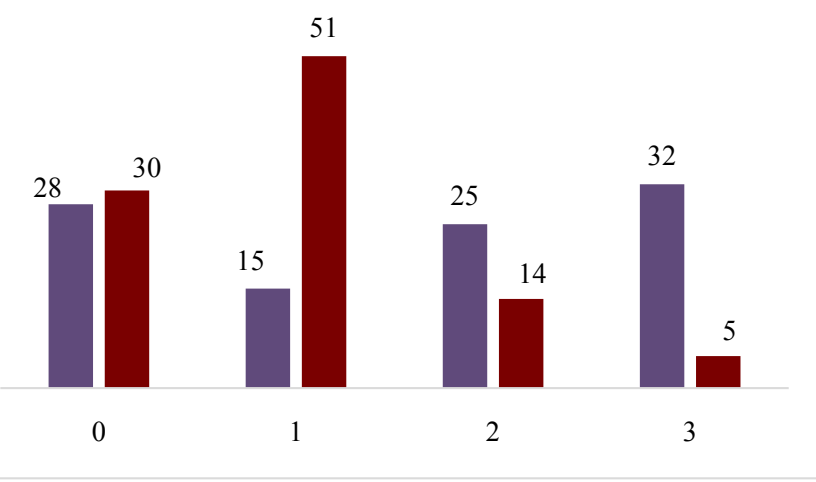

Figure 3. CRT performance (\%) versus final choice, Blue font: Laptop A; Red font: Laptop B.

Table 2. Personality traits.

\begin{tabular}{ccccccc}
\hline Degree, $\%$ & $\mathrm{H}$ & $\mathrm{E}$ & $\mathrm{X}$ & $\mathrm{A}$ & $\mathrm{C}$ & $\mathrm{O}$ \\
\hline Low & $35.6_{83}$ & $32.2_{75}$ & $74.7_{174}$ & $89.3_{208}$ & $34.8_{81}$ & $60.9_{142}$ \\
High & $64.4_{150}$ & $67.8_{158}$ & $25.3_{59}$ & $10.7_{25}$ & $65.2_{152}$ & $39.1_{91}$ \\
\hline
\end{tabular}

Note: Subscripts show the number of respondents. 
Then, we estimated the probability of abandonment of the prior choice by considering the mediating role of cognitive ability and personality. From the 233 participants, 224 picked Laptop A as their prior choice. Here, we considered only this subgroup of 224 participants, from which 94 switched to Laptop B. Thus, estimating the probability of abandonment of the prior choice here means switching from Laptop A to Laptop B.

Let $S$ be a dummy variable that takes the value 0 or 1 to indicate how preference strength is expected to shift the outcome. The state of no switching from Laptop A is represented by $S=0$, while $S=1$ is the state of switching from Laptop A to Laptop B. To obtain a transition probability from State 0 to State 1, we first estimate the linear predictor of switching from Laptop A to Laptop B:

$$
\eta=\beta_{0}+\beta_{1} S+\beta_{2} \mathrm{CRT}+\beta_{3} \mathrm{H}+\beta_{4} \mathrm{E}+\beta_{5} \mathrm{X}+\beta_{6} \mathrm{~A}+\beta_{7} \mathrm{C}+\beta_{8} \mathrm{O}+\varepsilon,
$$

where $\varepsilon$ is a random error. The transition probability from State 0 to State 1 can be estimated by a logistic model:

$$
P(\eta)=\frac{\exp \eta}{1+\exp \eta} .
$$

Table 3 shows the estimation of coefficients $\beta$ by the Fisher scoring method, which is equivalent to iteratively reweighted least squares.

Because variables agreeableness and openness to experience were statistically non-significant, Table 4 shows new estimates after dropping A and $\mathrm{O}$.

Table 4 shows that high preference strength is associated with a low probability of switching. This replicates the traditional result within the framework of Irmak and coauthors [5]. So, we find that in choices under incomplete information on incumbents, consumers with stronger preferences are less likely to abandon their prior choices. Motivated reasoning overrides cognitive dissonance.

However, our approach shows the conditions in terms of cognitive ability and personality traits for Irmak and coauthors to have a point. Table 4 also shows that low scores on the CRT increase the probability of switching from Laptop A to Laptop B. Thus, participants with lower cognitive ability are also more fickle. Table 4 shows, too, that the probability of switching from Laptop A to Laptop B increases for those with high emotionality and low honesty-humility, extraversion and conscientiousness. Thus, we also find that cognitive dissonance overrides motivated reasoning for those consumers with lower cognitive ability, and who are more emotional, less humble, less extraverted and less conscientious.

Using the results in Table 4, the linear predictor of switching from Laptop A to Laptop B is given by:

$$
\eta=13.2-0.6 S-0.87 C R T-1.02 H+1.58 E-1.48 X-1.18 C \text {. }
$$

This can be used to estimate the transition probability $P(\eta)$ from State 0 (no switching from Laptop A) to State 1 (switching from Laptop A to Laptop B) for each individual participant in our experiment. Figure 4 shows $\eta$ to participants who switched (in red) and to those who did not (in blue). The larger $\eta$, the bigger $P(\eta)$, and vice versa. 
To evaluate the fit of our model to the data, we consider that if $P(\eta)>0.5$ then one individual participant would switch from Laptop A to Laptop B; if $P(\eta)<0.5$ she would not. Table 5 shows the model adjusts well in 63 percent of cases (that is, $0.41+0.22$ ), which refer to the tails of the distribution in Figure 4. Of note, $P(\eta)$ increases for greater $\eta$, and values of $\eta$ approaching zero mean random switches, that is, $P(\eta)=0.5$.

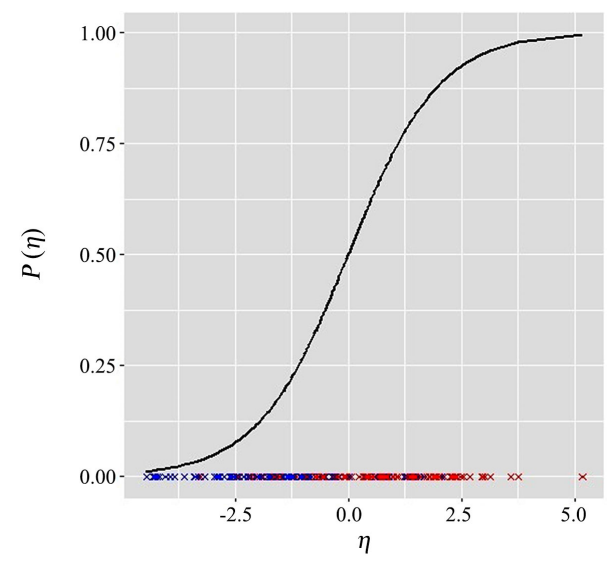

Figure 4. Probability distribution of switching from the prior choice. It shows the linear predictor of switching, $\eta$, to participants who switched from Laptop A to Laptop B (in red) and to those who did not (in blue). The larger $\eta$, the bigger $P(\eta)$, and vice versa.

Table 3. Estimate results for coefficients $\beta$.

\begin{tabular}{ccccc}
\hline Variable & Estimate & Standard error & Z value & $\mathrm{P}(>|z|)$ \\
\hline Intercept & 14.2594 & 3.2192 & 4.430 & $9.44 \mathrm{e}-06^{* * *}$ \\
$S$ & -0.5946 & 0.1553 & -3.829 & $0.000129^{* * *}$ \\
CRT & -0.8922 & 0.2101 & -4.246 & $2.18 \mathrm{e}-05^{\star * *}$ \\
H & -0.9522 & 0.3667 & -2.597 & $0.009403^{\star *}$ \\
E & 1.6039 & 0.4178 & 3.839 & $0.000123^{\star * *}$ \\
X & -1.3549 & 0.3691 & -3.671 & $0.000242^{* * *}$ \\
A & -0.4163 & 0.4378 & -0.951 & 0.341657 \\
C & -1.0759 & 0.4645 & -2.316 & $0.020544^{*}$ \\
O & -0.2641 & 0.3672 & -0.719 & 0.472025 \\
\hline
\end{tabular}

${ }^{*}$ significance between 0.01 and $0.05,{ }^{* *}$ significance between 0.001 and $0.01,{ }^{* * *}$ significance $<0.001$.

Table 4. Estimate results for significant $\beta$.

\begin{tabular}{ccccc}
\hline Variable & Estimate & Standard error & Z value & $\mathrm{P}(>|z|)$ \\
\hline Intercept & 13.1620 & 3.0532 & 4.311 & $1.63 \mathrm{e}-05^{* * *}$ \\
$S$ & -0.6027 & 0.1546 & -3.899 & $9.66 \mathrm{e}-05^{\star * *}$ \\
CRT & -0.8706 & 0.2077 & -4.191 & $2.78 \mathrm{e}-05^{* * *}$ \\
H & -1.0212 & 0.3581 & -2.851 & $0.004354^{* *}$ \\
E & 1.5784 & 0.4118 & 3.833 & $0.000126^{* * *}$ \\
X & -1.4836 & 0.3505 & -4.233 & $2.31 \mathrm{e}-05^{* * *}$ \\
C & -1.1763 & 0.4434 & -2.653 & $0.007981^{* *}$ \\
\hline
\end{tabular}

${ }^{* *}$ significance between 0.001 and $0.01,{ }^{* * *}$ significance $<0.001$. 
Table 5. Probability of switching from the prior choice.

\begin{tabular}{ccc}
\hline State & 0 & 1 \\
\hline 0 & 0.4107 & 0.1696 \\
1 & 0.1964 & 0.2232 \\
\hline
\end{tabular}

\section{Conclusions}

It seemed to be established in the literature that in choices under incomplete information on incumbents, consumers with stronger preferences are more likely to reinforce their prior choice. However, it has been suggested more recently that in situations where incomplete information is restricted only to the prior choice, consumers with stronger preferences are more likely to abandon, not reinforce, their prior choice. Motivated reasoning and cognitive dissonance can explain the contrasting results. The traditional result stands when motivated reasoning overcomes cognitive dissonance. The challenging result occurs when the reverse is true.

Here, we set an experiment to explicitly treat motivated reasoning and cognitive dissonance. For this purpose, we consider gauges of cognitive ability and personality traits to mediate the debate. We argued that those with a stronger System 2 are more likely to engage in motivated reasoning to reinforce their prior choice and thus suffer less cognitive dissonance, a rationale that could not be dismissed in our study.

Our results showed that high preference strength is associated with low probability of switching from brand A to brand B. This replicated the traditional result: motivated reasoning overcame cognitive dissonance. Thus, we found that in choices under incomplete information on incumbents, consumers with stronger preferences are less likely to abandon their prior choice.

However, we also found that cognitive dissonance overcame motivated reasoning for those consumers with lower cognitive ability, and who are more emotional, less humble, less extraverted, and less conscientious. Consumers with such characteristics are more likely to abandon their prior choice, even if they also held stronger prior preferences on incumbents.

\section{Acknowledgements}

Financial support from CNPq and Capes is acknowledged.

\section{Conflicts of Interest}

The authors declare no conflicts of interest regarding the publication of this paper.

\section{References}

[1] Kardes, F.R., Posavac, S.S., Silvera, D., Cronley, M.L., Sanbonmatsu, D.M., Schertzer, S., Miller, F., Herr, P.M. and Chandrashekaran, M. (2006) Debiasing Omission Neglect. Journal of Business Research, 59, 786-792. 
https://doi.org/10.1016/j.jbusres.2006.01.016

[2] Kunda, Z. (1990) The Case for Motivated Reasoning. Psychological Bulletin, 108, 480-498. https://doi.org/10.1037//0033-2909.108.3.480

[3] Pyszczynski, T. and Greenberg, J. (1987) Toward an Integration of Cognitive and Motivational Perspectives on Social Inference: A Biased Hypothesis Testing Model. Advances in Experimental Social Psychology, 20, 297-340. https://doi.org/10.1016/S0065-2601(08)60417-7

[4] Russo, J.E., Medvec, V.H. and Meloy, M.G. (1996) The Distortion of Information During Decisions. Organizational Behavior and Human Decision Processes, 66, 102-110. https://doi.org/10.1006/obhd.1996.0041

[5] Irmak, C., Kramer, T. and Sen, S. (2017) Choice under Incomplete Information on Incumbents: Why Consumers with Stronger Preferences Are More Likely to Abandon their Prior Choices. Journal of Consumer Psychology, 27, 264-269. https://doi.org/10.1016/j.jcps.2016.06.002

[6] Harmon-Jones, E. and Mills, J. (1999) Cognitive Dissonance: Progress on a Pivotal Theory in Social Psychology. American Psychological Association, Washington DC. https://doi.org/10.1037/10318-000

[7] Frederick, S. (2005) Cognitive Reflection and Decision Making. Journal of Economic Perspectives, 19, 25-42. https://doi.org/10.1257/089533005775196732

[8] De Vries, R.W. (2013) The 24-Item Brief HEXACO Inventory (BHI). Journal of Research in Personality, 47, 871-880. https://doi.org/10.1016/j.jrp.2013.09.003

[9] Evans, J.S.B.T. (2003) In Two Minds: Dual-Process Accounts of Reasoning. Trends in Cognitive Sciences, 7, 454-459. https://doi.org/10.1016/j.tics.2003.08.012

[10] Evans, J.S.B.T. (2008) Dual-Processing Accounts of Reasoning, Judgment, and Social Cognition. Annual Review of Psychology, 59, 255-278. https://doi.org/10.1146/annurev.psych.59.103006.093629

[11] Goldberg, L.R. (1993) The Structure of Phenotypic Personality Traits. American Psychologist, 48, 26-34. https://doi.org/10.1037//0003-066X.48.1.26

[12] McCrae, R.R. and Costa Jr, P.T. (2004) A Contemplated Revision of the NEO Five-Factor Inventory. Personality and Individual Differences, 36, 587-596. https://doi.org/10.1016/S0191-8869(03)00118-1

[13] Ashton, M.C., Lee, K., Perugini, M., Szarota, P., De Vries, R.E., Di Blas, L., Boies, K. and De Raad, B. (2004) A Six-Factor Structure of Personality-Descriptive Adjectives: Solutions from Psycholexical Studies in Seven Languages. Journal of Personality and Social Psychology, 86, 356-366. https://doi.org/10.1037/0022-3514.86.2.356

[14] Toplak, M.E., West, R.F. and Stanovich, K.E. (2014) Assessing Miserly Information Processing: An Expansion of the Cognitive Reflection Test. Thinking \& Reasoning, 20, 147-168. https://doi.org/10.1080/13546783.2013.844729 